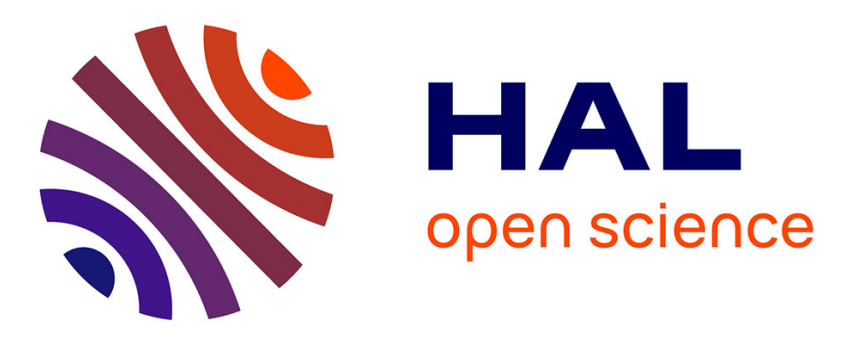

\title{
Effect of adding a third polysaccharide on the adsorption of protein microgels at the interface of polysaccharide-based water in water emulsions
}

\author{
João P E Machado, Lazhar Benyahia, Taco Nicolaï
}

\section{To cite this version:}

João P E Machado, Lazhar Benyahia, Taco Nicolaï. Effect of adding a third polysaccharide on the adsorption of protein microgels at the interface of polysaccharide-based water in water emulsions. Journal of Colloid and Interface Science, 2021, 603, pp.633-640. 10.1016/j.jcis.2021.06.053 . hal03357453

\section{HAL Id: hal-03357453 \\ https://hal.science/hal-03357453}

Submitted on 30 Sep 2021

HAL is a multi-disciplinary open access archive for the deposit and dissemination of scientific research documents, whether they are published or not. The documents may come from teaching and research institutions in France or abroad, or from public or private research centers.
L'archive ouverte pluridisciplinaire HAL, est destinée au dépôt et à la diffusion de documents scientifiques de niveau recherche, publiés ou non, émanant des établissements d'enseignement et de recherche français ou étrangers, des laboratoires publics ou privés. 


\section{Effect of adding a third polysaccharide on the adsorption of protein microgels at the interface of polysaccharide-based water in water emulsions}

João P. E. Machado ${ }^{\mathrm{a}, \mathrm{b}}$, Lazhar Benyahia ${ }^{\mathrm{b}}$, and Taco Nicolai ${ }^{\mathrm{b}}$

a BioPol, Chemistry Department, Federal University of Paraná, 81.531-980 Curitiba, Paraná, Brazil.

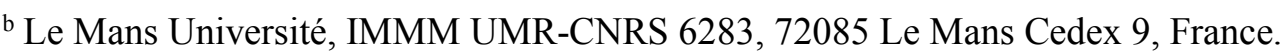

Corresponding author Taco Nicolai: tel: (33)-0243833139; email: Taco.Nicolai@univ-lemans.fr João P. E. Machado:31joaopedro@gmail.com

Lazhar Benyahia: lazhar.benyahia@univ-lemans.fr 


\begin{abstract}
Hypothesis

Water-in-water (W/W) emulsions are formed by mixing aqueous solutions of incompatible polymers and can in some cases be stabilized by addition of particles. The adsorption of particles at the interface of $\mathrm{W} / \mathrm{W}$ emulsions is dictated by the interfacial tension between the two aqueous phases and between the particles and each phase. It should therefore be possible to induce and fine-tune adsorption by adding small amounts of a third polysaccharide that is compatible with one or both phases.

Experiments

W/W emulsions were formed by mixing aqueous solutions of pullulan (PUL), amylopectin (AMP), and protein microgels (MG). The microstructure and positioning of the MG were monitored using confocal laser scanning microscopy. The effect of adding small amounts of other types of polysaccharides on the adsorption of the $\mathrm{MG}$ at the interface and their partitioning between the phases was studied.

\section{Findings}

The addition of all polysaccharides led to a progressive shift of the MG from the PUL phase to the AMP phase and to adsorption of the MG at the interface when the partition was not extreme. The partition could be fine-tuned to be equal, in which case particles adsorbed at the interface even very close to the binodal. The findings were confirmed for another type of emulsion and particle.
\end{abstract}

Keywords: Water-in-water emulsion; Pickering emulsion; interfacial tension; protein microgels; aqueous two-phase system 


\section{Introduction}

Mixing two thermodynamically incompatible polymers in water gives rise to aqueous two-phase systems $[1,2]$. When the two aqueous phases are mechanically mixed, they form waterin-water (W/W) emulsions similar to mixing oil and water. However, W/W emulsions have different properties when compared to conventional oil and water $(\mathrm{O} / \mathrm{W})$ emulsions. The width of the interface between two aqueous polymer solutions is wider than that between oil and water. Also, since both phases are aqueous solutions, the interfacial tension in $\mathrm{W} / \mathrm{W}$ emulsions is orders of magnitude lower [3,4]. Therefore, molecular surfactants cannot stabilize W/W emulsions, but solid particles [5-7] and, more recently, linear homo-polyelectrolytes [8] have shown to be efficient stabilizers in some cases. The stabilization mechanism is the same as for $\mathrm{O} / \mathrm{W}$ emulsions in the presence of particles known as Pickering emulsions $[9,10]$ and results from a reduction of the free energy when a particle adsorbs at the interface between the two phases A and B:

$\left.\Delta G=-\pi R^{2} \gamma_{A B}(1-\mid \cos \theta) \mid\right)^{2}$

where $R$ is the particle radius, $\gamma_{A B}$ is the interfacial tension between the liquid phases, and $\theta$ is the contact angle between the particles and the interface. The contact angle is determined by the difference between the interfacial tension of the particles with phase $\mathrm{A}\left(\gamma_{\mathrm{PA}}\right)$ and with phase $\mathrm{B}$ $\left(\gamma_{\mathrm{PB}}\right)$ as follows:

$$
\cos (\theta)=\frac{\left(\gamma_{P A}-\gamma_{P B}\right)}{\gamma_{A B}}
$$

which implies that particles spontaneously adsorb at the interface only if $\gamma_{\mathrm{AB}}>\left|\gamma_{\mathrm{PA}}-\gamma_{\mathrm{PB}}\right|$. The difficulty is therefore to find particles with suitable properties so that they adsorb at the interface of a given $\mathrm{W} / \mathrm{W}$ emulsion. An alternative to searching for particles with a suitable surface chemistry is to modify the surface of the particles. The latter method was demonstrated by Gonzalez et al. [11], who physically adsorbed different quantities of proteins at the surface of latex particles. By fine-tuning the quantity of adsorbed proteins, the stability of a W/W emulsion could be optimized. In this study, we have explored another alternative approach by adding a small amount of a third polymer that is miscible with the two aqueous phases and does not interact with the particles. In this manner, the interfacial tensions between the particles and each phase can be fine-tuned to favor adsorption of the particles at the interface.

A not yet explored system was investigated composed of aqueous solutions of amylopectin (AMP) and pullulan (PUL) that produced biphasic mixtures. AMP is the major component of starch and is a highly branched structure formed by $\alpha$-D-glucopyranose $(1 \rightarrow 4)$ with $\alpha$-D-glucopyranose $(1 \rightarrow 6)$ links [12]. PUL is a linear microbial polymer produced by a yeast-like fungus and consist of maltotriose repeat units connected by $\alpha$-maltotriose $(1 \rightarrow 6)$ 
glycosidic linkages[13]. To these mixtures, protein microgels (MG) were added that were produced by heating whey protein isolate (WPI) solutions at a $\mathrm{pH}$ close to the isoelectric point of the protein $(\mathrm{pH} \sim 5.0)$. MG have been used in the past to stabilize some types of $\mathrm{W} / \mathrm{W}$ emulsions [14-17]. However, in many W/W systems the MG do not adsorb at the interface but partition to one of the phases. In addition, even if the particles adsorb at the interface at high polymer concentrations, they no longer do so closer to the binodal, when $\gamma_{\mathrm{AB}}$ becomes very small. It will be shown here that the addition of a small amount of a third polysaccharide that does not interact specifically with any of the other components can modify the partition of the MG between the phases and can lead to adsorption of MG at the interface. The phenomenon was observed both for anionic polysaccharides (pectin, alginate, and $\kappa$-carrageenan) and a neutral polysaccharide (xyloglucan). In order to check the generality of the findings, a few measurements were done with mixtures of dextran and AMP in the presence of MG and with mixtures of PUL and AMP in the presence of cellulose nanocrystals. These measurements allow us to conclude that addition of a third polymer can be a general method to drive particles to the interface in $\mathrm{W} / \mathrm{W}$ emulsions and to improve their stability.

\section{Materials and methods}

\section{Materials}

Dextran (DX), alginate (ALG), amylopectin (AMP) from maize and $\kappa$-carrageenan (KC) were purchased from Sigma-Aldrich. Low-methylated pectin (PEC) from lemon peel was kindly provided by Cargill. Pullulan (PUL) and xyloglucan (XG) were kindly provided by Hayashibara co. and DSP Gokyo Food \& Chemical co., respectively. DX and PUL were used as received. Both PEC, $\mathrm{XG}$, and ALG solutions were centrifuged to remove insoluble aggregates. In order to remove potassium ions from the $\mathrm{KC}, 0.5 \mathrm{wt} \%$ solutions were dialyzed first against $0.1 \mathrm{~mol} \mathrm{~L}^{-1}$ $\mathrm{NaCl}$ and then extensively against ultrapure water. The $\mathrm{KC}$ was subsequently freeze-dried. Amylopectin (AMP) from maize was purchased from Sigma-Aldrich and was purified before use by adapting a method described in ref. [18]. Briefly, a $5 \mathrm{wt} \%$ AMP solution was dispersed in a mixture of dimethyl sulphoxide (DMSO) and water 95:5 v/v. Insoluble material was removed by centrifugation. The AMP in the supernatant was precipitated by adding 3 volumes of ethanol and subsequently rinsed with acetone and diethyl ether. The precipitate was dried under vacuum for 2 days at $30^{\circ} \mathrm{C}$. The polysaccharides were characterized by size-exclusion chromatography with on-line light scattering detection and the weight-average molar masses $\left(\mathrm{M}_{\mathrm{w}}\right)$ were the following: $\mathrm{DEX}=4.1 \times 10^{5}, \mathrm{ALG}=7.9 \times 10^{4}, \mathrm{KC}=8.8 \times 10^{4}, \mathrm{PEC}=3.7 \times 10^{6}, \mathrm{PUL}=3.0 \times 10^{5}, \mathrm{XG}=1.0$ $\times 10^{6}$, and $\mathrm{AMP}=1.6 \times 10^{8} \mathrm{~g} \mathrm{~mol}^{-1}$. 
Protein microgels (MG) were obtained following a method described in ref. [19]. Whey protein isolate (WPI) (BiPRO $\left.{ }^{\circ}\right)$ containing $93 \mathrm{wt} \%$ protein was purchased from Davisco Foods International, Inc. (Le Sueur, MN, USA). It was solubilized in water at approximately $10 \mathrm{wt} \%$ and centrifuged. The supernatant was subsequently filtrated through $0.4 \mu \mathrm{m}$ and $0.2 \mu \mathrm{m}$ pores, after which the concentration was adjusted to $4 \mathrm{wt} \%$ and the $\mathrm{pH}$ was set to 5.9 before heating for $15 \mathrm{~h}$ at $80^{\circ} \mathrm{C}$. The MG were analysed by light scattering and the resulting hydrodynamic radius $\left(\mathrm{R}_{\mathrm{h}}\right)$, radius of gyration $\left(\mathrm{R}_{\mathrm{g}}\right)$, and $\mathrm{M}_{\mathrm{w}}$ were $120 \mathrm{~nm}, 125 \mathrm{~nm}$, and $3.30 \times 10^{8} \mathrm{~g} / \mathrm{mol}$. Centrifugation showed that approximately $65 \%$ of the WPI was transformed into MG, with the residual forming small strand-like aggregates that do not adsorb to $\mathrm{W} / \mathrm{W}$ interfaces.

Cellulose nanocrystals (CNC) (CAS number: 9004-34-6) were purchased from the University of Maine's Process Development Center (USA). The powder was dispersed in Millipore water by ultrasonication using a sonicator from Bioblock Scientific. The molar mass $(\mathrm{Mw})$, the radius of gyration $(\mathrm{Rg})$, and the hydrodynamic radius $\left(\mathrm{R}_{\mathrm{h}}\right)$ of the $\mathrm{CNC}$ were determined using static and dynamic light scattering as described in ref. [20]: $\mathrm{M}_{\mathrm{w}}=8.6 \times 10^{6} \mathrm{~g} / \mathrm{mol}, \mathrm{R}_{\mathrm{g}}=52$ $\mathrm{nm}$ and $\mathrm{R}_{\mathrm{h}}=37 \mathrm{~nm}$.

Emulsions were prepared by adding together aqueous stock solutions of the various ingredients in the required amounts, followed by mixing under a vortex. No effect was seen of the order of mixing nor of the duration of mixing.

\section{Methods}

The phase diagram of the AMP-PUL mixture was established based on macroscopic observations. Tubes containing different quantities of the stock solutions of AMP (15 wt \%) and PUL (30 wt \%) were mixed under a vortex, after which they were checked for macroscopic phase separation that was accelerated by centrifugation. Water was then added to the mixtures of different compositions until phase separation was no longer observed, in order to determine the binodal. When phase separation was almost complete, the relative heights of each phase allowed determination of the tie-lines also considering the amount of PUL that remained in the AMP phase that was deduced from the binodal.

Confocal Laser Scanning Microscopy (CLSM) images were obtained with a Zeiss LSM800 (Carl Zeiss Microscopy GmbH) using water immersion objectives: HC $\times$ PL APO $63 \times$ $(\mathrm{NA}=1.2)$ and a $\mathrm{HC} \times \mathrm{PL} \mathrm{APO} 25 \times(\mathrm{NA}=0.7)$. The samples were either inserted into acrylic wells plates or hermetically-sealed concave slides covered with glass slips. The MG were physically labeled with rhodamine B at approximately $5 \mathrm{ppm}$. PEC was covalently labeled with fluorescein isothiocyanate (FITC) by adapting a method described in the ref. [21]. Briefly, $1 \mathrm{~g}$ of 
PEC was dispersed in $20 \mathrm{~mL}$ of DMSO followed by the addition of $0.5 \mathrm{~mL}$ of $\mathrm{HCl} 1 \mathrm{~mol} \mathrm{~L}^{-1}$. Then, $0.04 \mathrm{~g}$ of FITC and $40 \mu \mathrm{L}$ of dibutyltin dilaurate was added to the mixture, which was left stirring at $80^{\circ} \mathrm{C}$ for 2 days. The PEC-FITC was then precipitated and washed with ethanol $99 \%$ until the solvent became colorless. The precipitate was subsequently dissolved in water and dialyzed against Milli-Q water to remove any residual free FITC. A few insoluble aggregates of PEC-FITC formed during the labeling process were observed in the experiments, but they did not interfere with the system. The fluorescence of FITC was exited at $480 \mathrm{~nm}$ and detected between 480 and $540 \mathrm{~nm}$. The fluorescence of rhodamine B was exited at $580 \mathrm{~nm}$ and detected between 580 and $800 \mathrm{~nm}$. We verified that at the conditions used here the fluorescence intensity was proportional to the concentration of the labeled polymers.

The partitioning of protein between the phases was quantified by measuring the extinction at a wavelength of $280 \mathrm{~nm}$ using a UV-visible spectrometer (Jasco PAC-743). The absorbance can be measured with an accuracy of about $\pm 2 \%$. Notice that there was no need here to know the extinction coefficient accurately because we were interested in the relative difference between the concentration in the two phases. After macroscopic phase separation, both the AMP and PULrich phase were analyzed at $\Phi_{\mathrm{PUL}}=0.13,0.50$, and 0.87 at different PEC concentrations and a fixed MG concentration of $0.4 \mathrm{wt} \%$.

\section{Results and discussion}

The phase diagram of AMP-PUL is shown in Erreur ! Source du renvoi introuvable.. The critical point was situated at $2.8 \mathrm{wt} \%$ AMP and $2.1 \mathrm{wt} \%$ PUL. W/W emulsions at two compositions were studied as indicated in Figure 1: $7.8 \mathrm{wt} \%$ AMP, $1.4 \mathrm{wt} \%$ PUL and $1.2 \mathrm{wt} \%$ AMP, $5.1 \mathrm{wt} \%$ PUL. The two compositions were situated on the same tie-line, which means that the interfacial tension between the AMP and PUL phase was the same, but had different volume fractions $(\Phi)$ of each phase. In one emulsion the PUL phase formed droplets with $\Phi=0.13$ in a continuous AMP phase (P/A) and in the other emulsion the AMP phase formed droplets with $\Phi=0.13$ in a continuous PUL phase (A/P). At the chosen compositions, the fraction of AMP in the PUL phase was negligible, but the AMP phase still contained about $0.8 \mathrm{wt} \%$ PUL. 


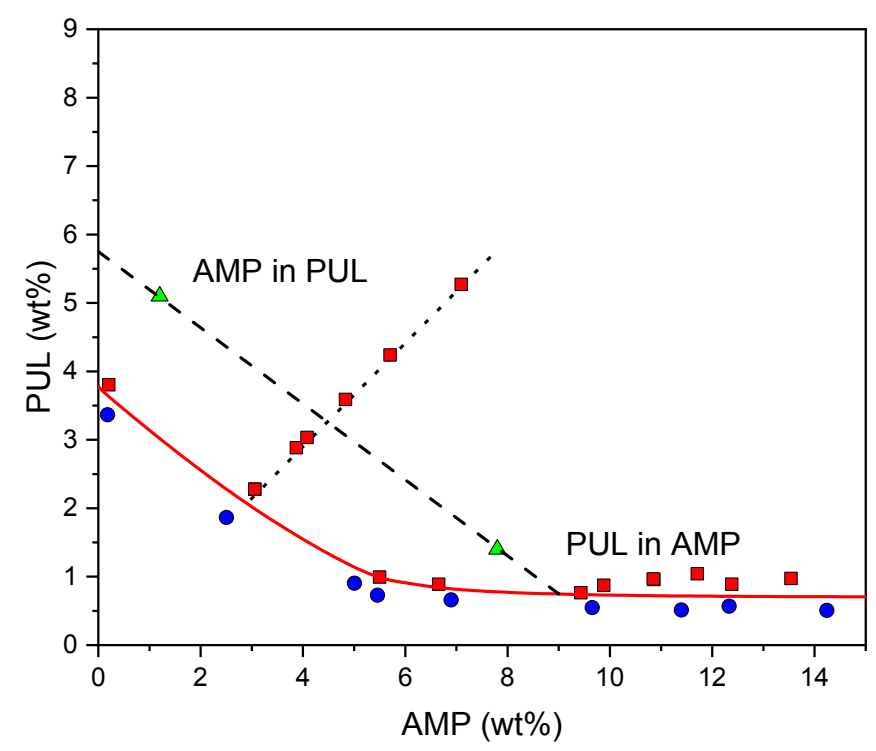

Figure 1. Phase diagram of aqueous mixtures of AMP and PUL. The binodal (solid) and the 50:50 volume fraction (dotted) lines are shown. The squares and circles represent phase-separated and homogeneous systems, respectively. The triangles indicate the compositions that were studied, and the dashed line shows the tie-line on which they are situated.

\section{Effect of the addition of pectin}

MG that were added to the emulsions did not adsorb at the interface, but were situated preferentially inside the PUL phase. When PEC was added to the mixtures at different concentrations, the MG partition changed, see Erreur! Source du renvoi introuvable.. With increasing PEC concentration, an increasing amount of MG adsorbed at the interface, and at higher PEC concentrations, excess microgels migrated to the AMP phase. These transitions were observed for both $\mathrm{A} / \mathrm{P}$ and $\mathrm{P} / \mathrm{A}$ emulsions but at different PEC concentrations $\left(\mathrm{C}_{\mathrm{PEC}}\right)$. When PUL was the continuous phase, the ring of adsorbed MG is clearly seen for $\mathrm{C}_{\mathrm{PEC}}>0.03 \mathrm{wt} \%$, whereas for $\mathrm{P} / \mathrm{A}$ emulsions, it is already clear for $\mathrm{C}_{\mathrm{PEC}} \geq 0.005 \mathrm{wt} \%$. Interestingly, the $\mathrm{MG}$ no longer adsorbed at the interface of $\mathrm{P} / \mathrm{A}$ and $\mathrm{A} / \mathrm{P}$ emulsions when $\mathrm{C}_{\mathrm{PEC}} \geq 0.03 \mathrm{wt} \%$ and $\mathrm{C}_{\mathrm{PEC}} \geq 0.1 \mathrm{wt} \%$, respectively, suggesting that when the preference of MG for the AMP became too strong, adsorption at the interface no longer reduced the total free energy. 


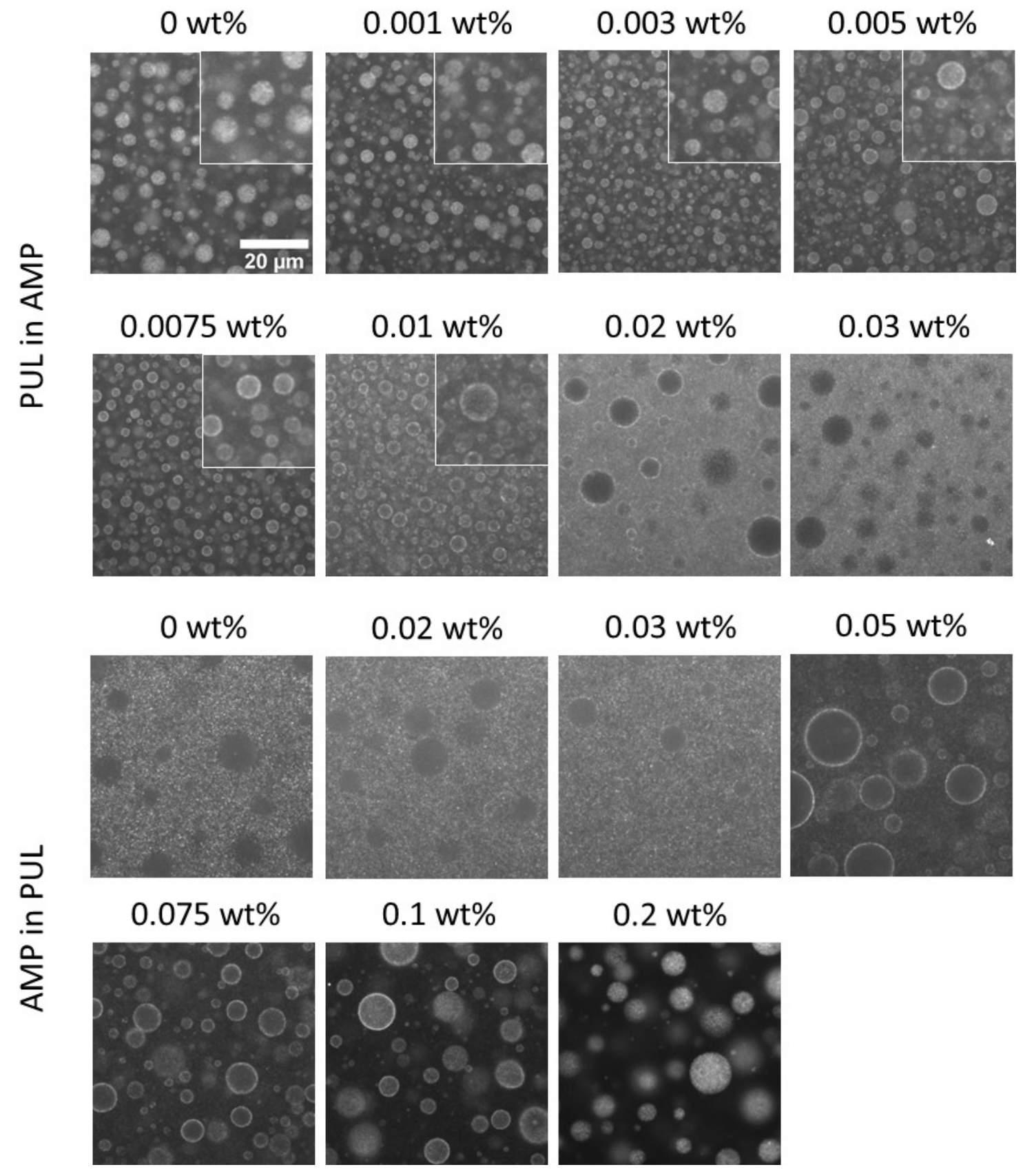

Figure 2. CLSM images of freshly prepared $P / A$ and A/P emulsions containing $0.4 w t \% M G$ and different PEC concentrations as indicated in the figure. The $M G$ were physically labeled with rhodamine B. All images are at the same scale. The inserts added to some images represent zooms (20 $20 \mu \mathrm{m})$ of a part of the main image.

The transition of the MG from the PUL phase to the AMP phase can also be observed visually after macroscopic phase separation as the suspended MG rendered the phase turbid, see Erreur! Source du renvoi introuvable. Notice that, whereas pure PUL solutions were transparent, pure AMP solutions were opalescent due to a small amount of large aggregates. Comparison of Figures 2 and 3, shows that there was a small influence of the labeling with rhodamine $\mathrm{B}$ on the partition of the MG which shifted the transition from between $0.075-0.01$ 
wt $\%$ to between $0.01-0.02 \mathrm{wt} \%$ for P/A. Curiously, this effect was much more pronounced in $\mathrm{P} / \mathrm{A}$ emulsions than in $\mathrm{A} / \mathrm{P}$ emulsions.

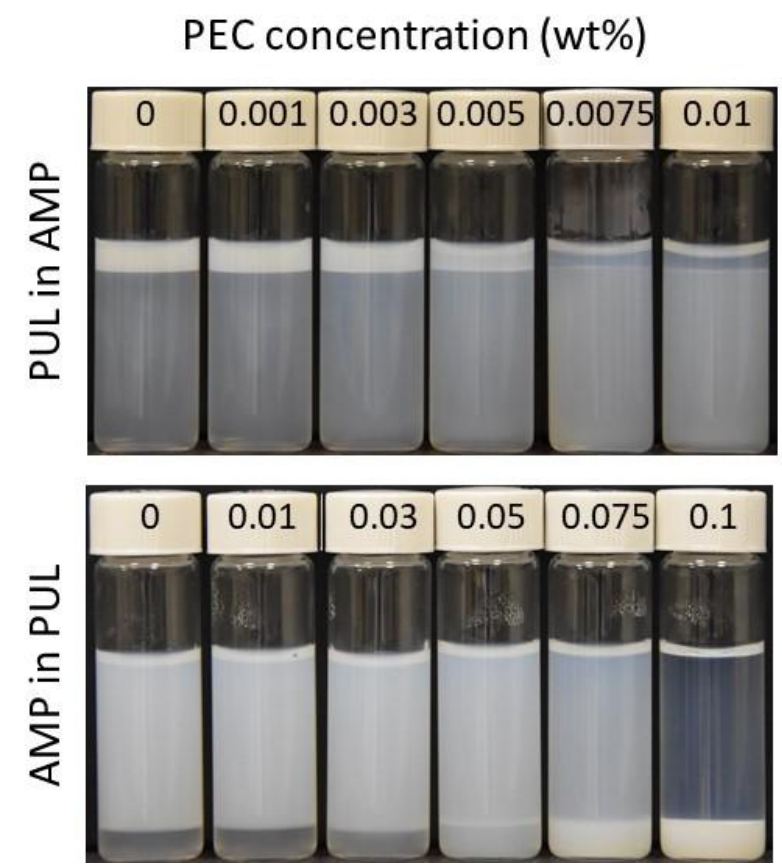

Figure 3. P/A and A/P emulsions after macroscopic phase separation containing $0.4 \mathrm{wt} \% \mathrm{MG}$ and different PEC concentrations as indicated in the figure.

The ratio (R) of the MG concentration in the AMP over that in the PUL phase was determined by measuring the absorbance of both the top PUL and bottom AMP phases at a wavelength of $278 \mathrm{~nm}$ in the absence of rhodamine B. Figure 4 shows the results as a function of the $\mathrm{PEC}$ concentration for $\mathrm{A} / \mathrm{P}$ and $\mathrm{P} / \mathrm{A}$ emulsions as well as for emulsions containing equal volume fractions of both phases at the same tie-line. The MG partitioned strongly to the PUL phase in the absence of PEC and migrated progressively to the AMP with increasing PEC concentration. The strong partition of the MG contrasts with the almost equal distribution of native WPI between the two phases independent of the polysaccharide concentration (results not shown). The implication is that the mixing entropy of the relatively small native proteins dominated the free energy gain by partitioning. As we noted in the materials section, the MG suspension contained a sizeable fraction $(\approx 35 \%)$ of small strand-like aggregates. These smaller aggregates also partitioned to different phases, as did the MG, even though most likely they did not adsorb at the interface. The transition occurred at higher PEC concentrations when the volume fraction of PUL $\left(\Phi_{\mathrm{PUL}}\right)$ was increased. Within the experimental uncertainty, which can be estimated from the spread of the data in Figure 4, the PEC concentration at which $R=1$ is 
proportional to $\Phi_{\text {PUL. If }}$ we assume that all pectin partitioned to the PUL phase, $R=1$ when the PUL phase contained approximately $0.07 \mathrm{wt} \%$ pectin.

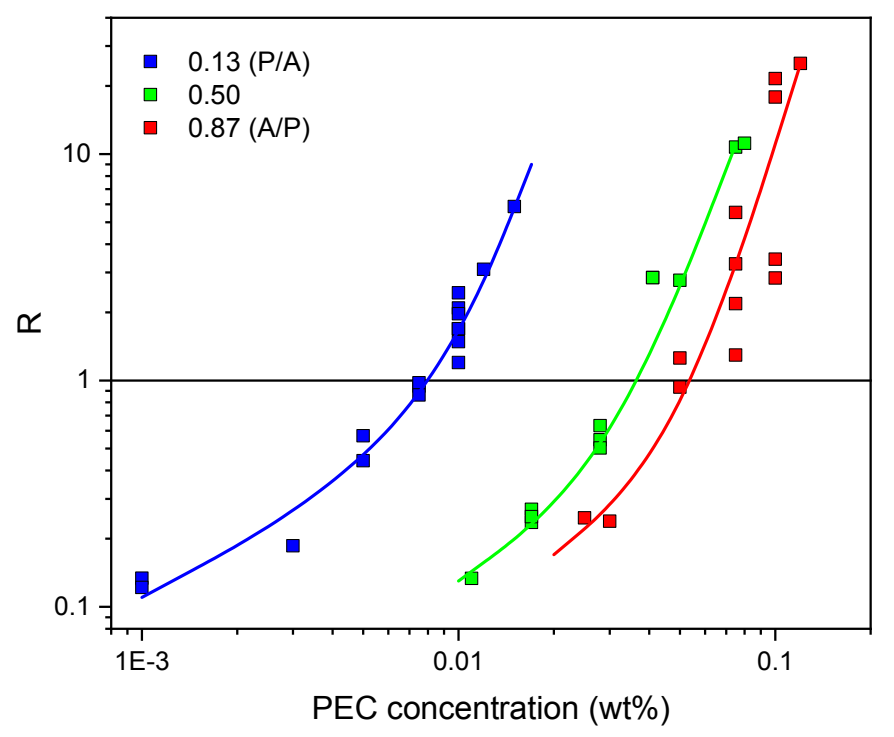

Figure 4. Ratio of the $M G$ concentrations $(R)$ in the AMP and PUL phases as a function of the $P E C$ concentration for emulsions at 3 different $\Phi_{P U L}$ as indicated in the figure. The solid lines are guides to the eye.

As was explained above, it is expected that MG spontaneously adsorb at the interface when $\gamma_{\text {PUL-AMP }}>\left|\gamma_{\text {MG-PUL }}-\gamma_{\text {MG-AMP }}\right|$. The observation that MG adsorb at the interface when PEC is added implies that $\gamma_{\text {PUL-AMP }}$ increased and/or $\left|\gamma_{\text {MG-PUL-}} \gamma_{\text {MG-AMP }}\right|$ decreased. Initially, adding PEC caused a decrease in the term $\left|\gamma_{\text {MG-PUL }} \gamma_{\text {MG-AMP }}\right|$ until $\gamma_{\text {MG-AMP }}=\gamma_{\text {MG-PUL }}$ at $\mathrm{R}=1$. Further increase

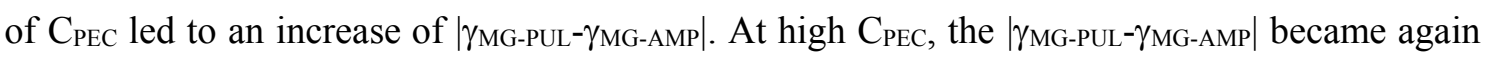
larger than $\gamma_{\text {PUL-AMP }}$ so that MG no longer adsorbed at the interface. Whatever is the effect of PEC on $\gamma_{\text {PUL-AMP, }}$ the fact that MG do not adsorb at high PEC concentrations shows that $\gamma_{\text {PUL-AMP }}$ does

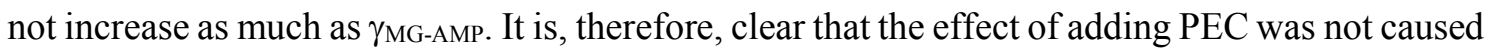
by a change in the interfacial tension between the two bulk phases, but by the reduction of the difference between $\gamma_{\text {MG-PUL }}$ and $\gamma_{\text {MG-AMP. }}$

When W/W emulsions are diluted, $\gamma_{\mathrm{AB}}$ decreases and becomes zero at the binodal. Generally, particles no longer adsorb at the interface at some point during progressive dilution because $\left|\gamma_{\mathrm{PA}-} \gamma_{\mathrm{PB}}\right|$ does not decrease as strongly with dilution as $\gamma_{\mathrm{AB}}$. However, if the system can be tuned such that $\left|\gamma_{\mathrm{PA}}-\gamma_{\mathrm{PB}}\right|=0$, the particles should remain at the interface as long as there is phase separation. We have tested this hypothesis by diluting an $\mathrm{A} / \mathrm{P}$ emulsion in the presence of 
$0.06 \mathrm{wt} \%$ PEC where $\mathrm{R} \approx 1$ and $\left|\gamma_{\mathrm{PA}-} \gamma_{\mathrm{PB}}\right|$ is close to zero and found that indeed MG remained at the interface even very close to the binodal (Figure S1). Similarly, one would expect that an increase of $\gamma_{\mathrm{AB}}$ by increasing the AMP or PUL concentration favors the adsorption of MG at the interface. This was confirmed by the finding that MG adsorbed at the interface even in the absence of PEC when the PUL concentration was increased above $8 \mathrm{wt} \%$.

It is remarkable that such small amounts of PEC can modify the partition of microgels. At $\mathrm{pH} 7.0$ association between PEC and MG is not expected since both possess negative net charge. To verify that MG and PEC did not form complexes, PEC was covalently labeled with FITC (FITC-PEC). Labeling of PEC and MG with different fluorophores allowed the distinction between the two in CLSM images of A/P emulsions, see Figure 5. Whereas the MG clearly adsorbed at the interface (Figure 5B), no accumulation of FITC-PEC at the interface can be seen (Figure 5A), showing that no complexation had occurred between PEC and MG. It was shown elsewhere, using the same methodology, that $\kappa$-carrageenan $(\mathrm{KC})$ forms complexes with MG only below pH 5.5 [14]. We found that complexes between MG and PEC also started to be formed only below $\mathrm{pH} 5.5$.

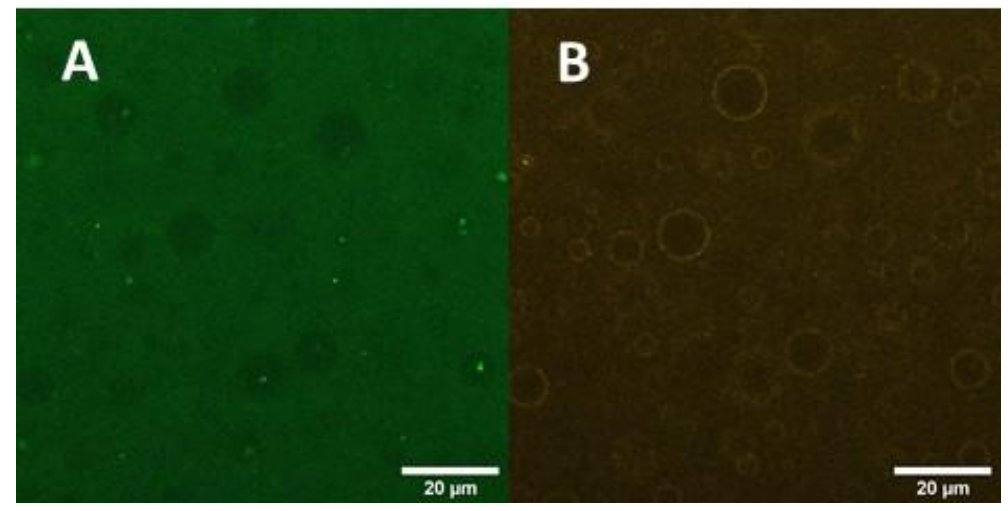

Figure 5. CLSM images of the same field-of-view of $A / P\left(\Phi_{P U L}=0.87\right)$ emulsion containing 0.4 $w t \% M G$ and $0.05 w t \%$ FITC-PEC. The fluorescence signal of FITC (A) and that of rhodamine $B(B)$ are shown for the same image.

As we mentioned above, Figure 4 shows that if all PEC migrated to the PUL phase, the concentration of PEC at $\mathrm{R} \approx 1$ would be approximately $0.07 \%$ at the three $\Phi_{\mathrm{PUL}}$. However, when we compare the fluorescence intensity of FITC-PEC present in the two phases, we find that the PUL phase contained approximately only $20 \%$ more FITC-PEC at all tested volume fractions. It explains the relatively low contrast between the dispersed and continuous phase in Figure 5A, which we did not artificially enhance. One possible explanation is that labeling influenced partitioning. We checked for this possibility by measuring R with FITC-PEC and found that 
indeed the effect of FITC-PEC on the partitioning was much weaker than that of unlabelled PEC. The implication is that the fluorescence intensity distribution does not reflect a much stronger partitioning of unlabeled PEC. Unfortunately, it is very difficult to detect these relatively small PEC concentrations in the concentrated PUL phase by other techniques, and using higher PEC concentrations is likely to influence the partitioning.

Generalization to other systems

A similar effect on the partitioning of the MG was found when alginate (ALG) or $\kappa$ carrageenan $(\mathrm{KC})$ were added. In both cases, the addition of the anionic polysaccharides induced migration of the MG from the PUL phase towards the AMP phase and led to adsorption at the interface, see Figure 6. In fact, the effect occurred already at somewhat lower concentrations for ALG and $\mathrm{KC}$ than for PEC.

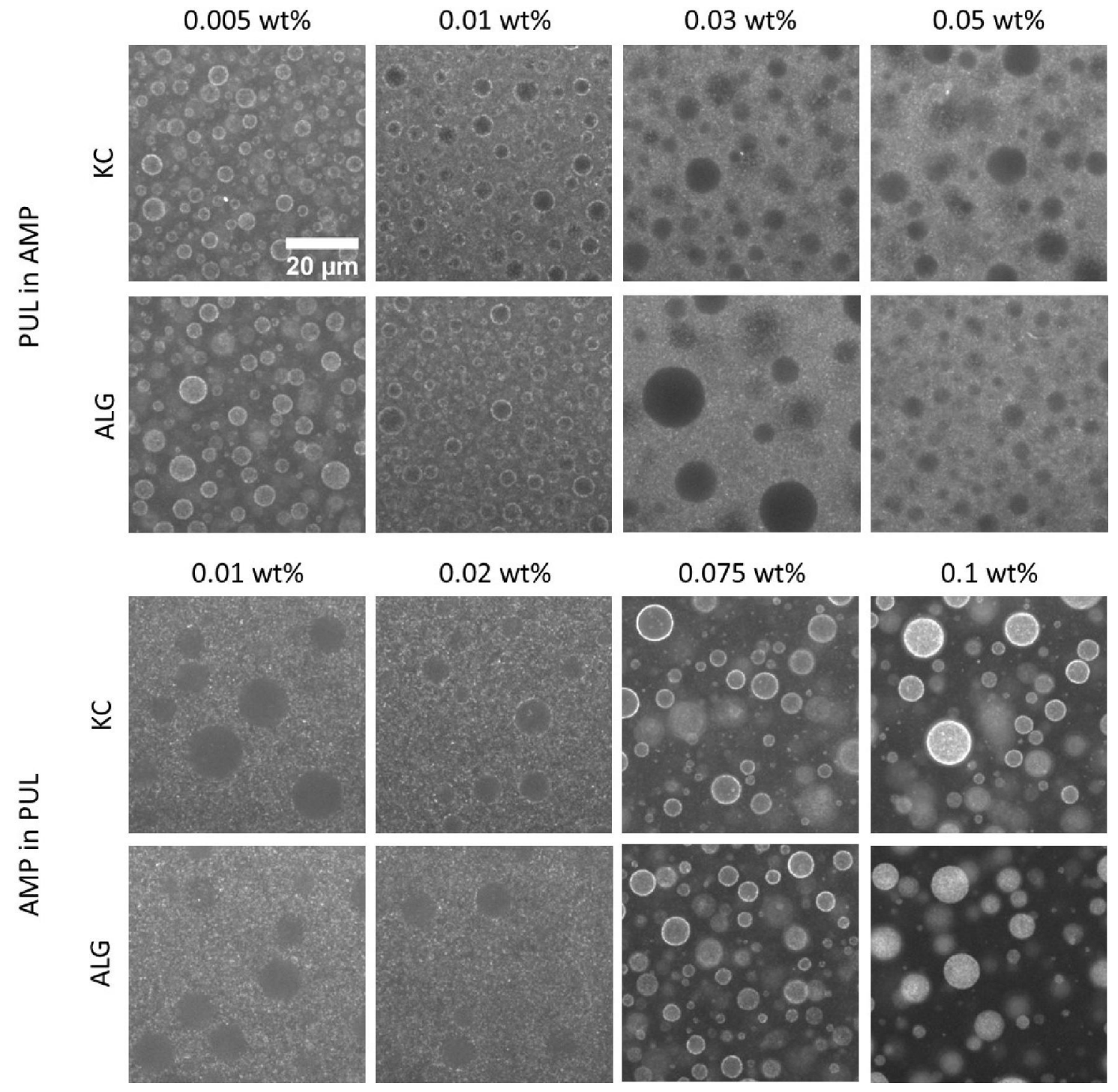


Figure 6. CLSM images of freshly prepared A/P and P/A emulsions in the presence of $0.4 w t \%$ $M G$ and different $A L G$ and $K C$ concentrations, as indicated in the figure. All images are at the same scale.

In order to test whether the effect is related to the charge on the added polysaccharides, the effect of adding the neutral polysaccharide xyloglucan (XG) was investigated. Similar phenomena were observed, but it required higher concentrations for XG than for the anionic polysaccharides. It was found that the transition of MG from PUL to AMP phase happened between 0.2 to $0.5 \mathrm{wt} \% \mathrm{XG}$ in $\mathrm{A} / \mathrm{P}$ emulsions and between 0.1 to $0.2 \mathrm{wt} \%$ in $\mathrm{P} / \mathrm{A}$ emulsions, see Figure S2 of the supplementary information. We noted that XG strongly prefers PUL over AMP. Actually, XG and AMP phase separate at higher concentrations, as was reported elsewhere [17]. Besides, MG partitioned to the AMP phase in phase-separated mixtures of XG and AMP, whereas they partitioned to the PUL in phase-separated mixtures of PUL/AMP. This indicates that when $\mathrm{XG}$ is added to mixtures of PUL/AMP, it enters the PUL phase and reduces the affinity of this phase for the MG.

The phenomenon discussed here for PUL/AMP mixtures was also observed for another type of W/W emulsion formed by mixing the weakly branched polysaccharide dextran (DEX) with AMP. For this system as well, MG did not adsorb at the interface but preferred to reside in the DEX phase. When PEC was added to the emulsions, MG migrated progressively to the AMP, see

Figure 7 and 8. More PEC was needed to induce the effect for DEX/AMP emulsions than for PUL/AMP emulsions, suggesting that the MG had a stronger preference for DEX than for PUL. This preference was confirmed by observing that MG partitioned to the DEX phase in a phase-separated mixture of DEX and PUL.

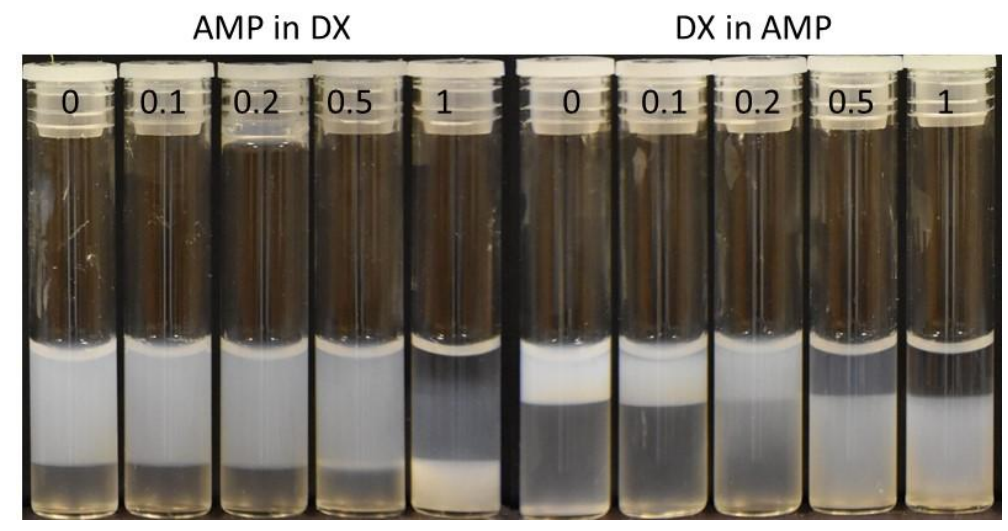

Figure 7. Macroscopic phase-separated mixtures of AMP in DX and DX in AMP emulsions with $0.4 w t \% M G$ at different PEC concentrations, as indicated in the figure. 

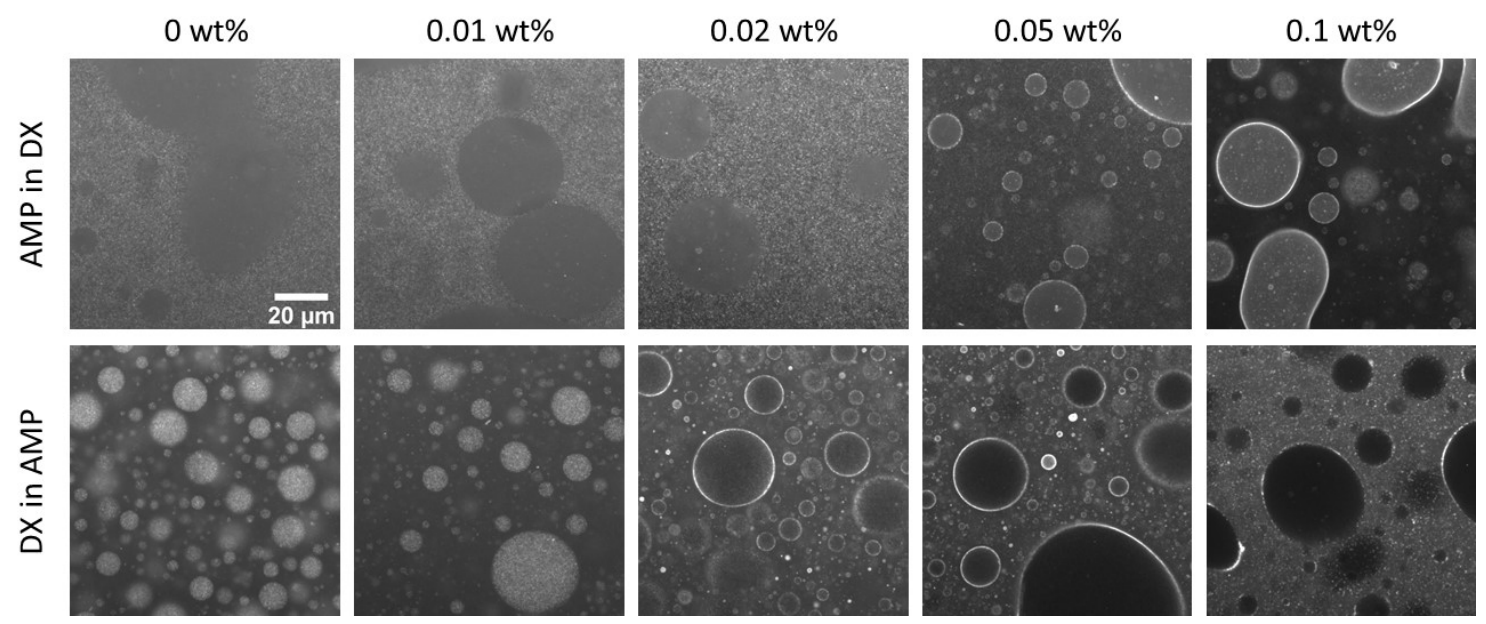

Figure 8. CLSM images of fresh emulsions of AMP and DEX in the presence of $0.4 \mathrm{wt} \% \mathrm{MG}$, and at different PEC concentrations as indicated in the figure. All images are at the same scale.

Finally, we found a similar effect of adding PEC for another type of particle, viz. cellulose nanocrystals (CNC) at $0.2 \mathrm{wt} \%$. These rod-like particles were reported to accumulate at the interface in W/W emulsions formed by mixing DEX and polyethylene oxide [22], but in the AMPPUL emulsions the CNC partitioned preferentially to the PUL phase and did not adsorb at the interface. However, when $0.11 \mathrm{wt} \%$ PEC was added the CNC partitioned to the AMP and could also faintly be observed at the interface, see Figure $\mathrm{S} 3$ of the supplementary information. When $0.18 \mathrm{wt} \%$ PEC was added the partitioning to the AMP phase was stronger and the CNC could no longer be observed at the interface.

It appears that the effect of adding a third polymer on the adsorption and partition of particles is quite general and not related to the charge or specific interactions. We believe that the effect is caused by changes in the interfacial tension between the particles and the phases. This change can be induced either by adding a polymer that has a higher affinity for the particle and that partitions to the phase that had lower affinity or by adding a component that has a lower affinity for the particles and that partitions to the phase that had a higher affinity for the particles. The latter was shown to be the case when XG was added to the AMP-PUL emulsion containing MG. By fine-tuning the addition of the component so that the particles partition equally to both phases, it is possible to maintain the particles at the interface even very close to the binodal.

It is by now well-established that the adsorption of particles at the interface is insufficient to procure stability against coalescence. In fact, none of the systems discussed here were stable at $\mathrm{pH}$ 7. However, at much higher AMP and PUL concentrations an emulsion at $\Phi_{\mathrm{PUL}}=0.87$ was found to be stable during at least one week in the presence of MG, but it destabilized within one day after dilution by a factor of 1.3 even though the MG were still adsorbed at the interface. No 
significant effect of adding ALG was found on the stability. In order to favor stability, one should introduce attractive interaction between the particles at the interface. Attractive interaction between MG can be induced by reducing the $\mathrm{pH}$, but this also leads to large-scale aggregation of MG within the bulk phases [23]. Large-scale aggregation can be avoided by the addition of anionic polysaccharides that bind to the $\mathrm{MG}$ at $\mathrm{pH}<5.5$ [14]. Elsewhere we will show that stable AMP-PUL emulsions can be obtained in this manner at low AMP and PUL concentrations by reducing the $\mathrm{pH}$ in the presence of well-chosen small amounts of anionic polysaccharide.

\section{Conclusions}

In the past, it has been shown that particles can adsorb at the interface between two aqueous phases, but only if the interfacial tension between the particles and each of the two phases is less than that between the two phases. Therefore one needs to either select particles of suitable chemistry or modify the surface of the particles. In this study, we tested a third approach, which had not yet been explored, i.e. to modify the interfacial tension between the particles and the phases by adding small amounts of a third polymer. The results reported here confirm the hypothesis that the adsorption of particles at the interface of water-in-water emulsions could be fine-tuned by adding a third polymer that mixes homogeneously with one or both phases. For the case of protein microgels added to $\mathrm{W} / \mathrm{W}$ emulsions formed by mixing amylopectin and pullulan, it was shown that for three anionic (ALG, PEC, and KC) and one neutral (XG) polysaccharide adding even a small amount led to a change in the partitioning of the MG between the phases favoring their adsorption at the interface even though the third polymer did not interact with the microgels. The origin of this phenomenon is that the added polymer modifies the interfacial tension between the particles and each of the two phases differently. When the interfacial tension between the particles and each phase is equal, adsorption of particles at the interface occurs even if the interfacial tension between the phases is extremely small, i.e. close to the binodal. The effect was also found for another emulsion formed by mixing amylopectin with dextran and for another type of particle (cellulose nanocrystals). More research is needed to understand the relationship between the chemistry of the third polymer and its effect on the adsorption of the particles at the interface. Another important aspect for future research is the effect of the interaction of the third polymer with the particles, which we will study for the present system by varying the $\mathrm{pH}$.

\section{Acknowledgement.}

This research was financed by the regional program Food for Tomorrow /Cap Aliment; Research, Education and Innovation in Pays de la Loire, which is supported by the French Region 
Pays de la Loire and the European Regional Development Fund. The CLSM analyses were conducted with the platform "Matière molle" at IMMM.

\section{Authors contributions:}

João Pedro Elias Machado: Investigation, writing - original draft;

Taco Nicolai: Supervision, conceptualization, data curation, writing - reviewing \& editing, funding acquisition

Lazhar Benyahia: Supervision, writing - reviewing \& editing, funding acquisition

\section{References}

[1] D. Gonzalez Ortiz, C. Pochat-Bohatier, J. Cambedouzou, M. Bechelany, P. Miele, Current Trends in Pickering Emulsions: Particle Morphology and Applications, Engineering. 6 (2020) 468-482. https://doi.org/10.1016/j.eng.2019.08.017.

[2] Y. Yang, Z. Fang, X. Chen, W. Zhang, Y. Xie, Y. Chen, Z. Liu, W. Yuan, An overview of pickering emulsions: Solid-particle materials, classification, morphology, and applications, Front. Pharmacol. 8 (2017) 1-20. https://doi.org/10.3389/fphar.2017.00287.

[3] H. Firoozmand, B.S. Murray, E. Dickinson, Interfacial structuring in a phase-separating mixed biopolymer solution containing colloidal particles, Langmuir. 25 (2009) 13001305. https://doi.org/10.1021/la8037389.

[4] E. Scholten, R. Tuinier, R.H. Tromp, H.N.W. Lekkerkerker, Interfacial tension of a decomposed biopolymer mixture, Langmuir. 18 (2002) 2234-2238. https://doi.org/10.1021/la0114373.

[5] E. Dickinson, Biopolymer-based particles as stabilizing agents for emulsions and foams, Food Hydrocoll. 68 (2017) 219-231. https://doi.org/https://doi.org/10.1016/j.foodhyd.2016.06.024.

[6] T. Nicolai, B. Murray, Particle stabilized water in water emulsions, Food Hydrocoll. 68 (2017) 157-163. https://doi.org/10.1016/j.foodhyd.2016.08.036.

[7] J. Esquena, Water-in-water (W/W) emulsions, Curr. Opin. Colloid Interface Sci. 25 (2016) 109-119. https://doi.org/10.1016/j.cocis.2016.09.010. 
[8] L. Tea, T. Nicolai, F. Renou, Stabilization of Water-In-Water Emulsions by Linear Homo-Polyelectrolytes, Langmuir. 35 (2019) 9029-9036.

https://doi.org/10.1021/acs.langmuir.9b01604.

[9] R. Aveyard, B.P. Binks, J.H. Clint, Emulsions stabilised solely by colloidal particles, Adv. Colloid Interface Sci. 100-102 (2003) 503-546. https://doi.org/10.1016/S00018686(02)00069-6.

[10] Y. Chevalier, M.A. Bolzinger, Emulsions stabilized with solid nanoparticles: Pickering emulsions, Colloids Surfaces A Physicochem. Eng. Asp. 439 (2013) 23-34. https://doi.org/10.1016/j.colsurfa.2013.02.054.

[11] A. Gonzalez-Jordan, T. Nicolai, L. Benyahia, Enhancement of the particle stabilization of water-in-water emulsions by modulating the phase preference of the particles, J. Colloid Interface Sci. 530 (2018) 505-510. https://doi.org/10.1016/j.jcis.2018.04.088.

[12] A. Buléon, P. Colonna, V. Planchot, S. Ball, Starch granules: Structure and biosynthesis, Int. J. Biol. Macromol. 23 (1998) 85-112. https://doi.org/10.1016/S01418130(98)00040-3.

[13] K. Nishinari, K. Kohyama, P.A. Williams, G.O. Phillips, W. Burchard, K. Ogino, Solution Properties of Pullulan, Macromolecules. 24 (1991) 5590-5593. https://doi.org/10.1021/ma00020a017.

[14] H. Khemissi, H. Bassani, A. Aschi, I. Capron, L. Benyahia, T. Nicolai, Exploiting Complex Formation between Polysaccharides and Protein Microgels To Influence Particle Stabilization of W/W Emulsions, Langmuir. 34 (2018) 11806-11813. https://doi.org/10.1021/acs.langmuir.8b02383.

[15] A. Gonzalez-Jordan, T. Nicolai, L. Benyahia, Influence of the Protein Particle Morphology and Partitioning on the Behavior of Particle-Stabilized Water-in-Water Emulsions, Langmuir. 32 (2016) 7189-7197. https://doi.org/10.1021/acs.langmuir.6b01993.

[16] B. Hazt, H.P. Bassani, J.P. Elias-Machado, J.L. Aldinucci Buzzo, J.L.M. Silveira, R.A. de Freitas, Effect of $\mathrm{pH}$ and protein particle shape on the stability of amylopectinxyloglucan water-in-water emulsions, Food Hydrocoll. (2020) 105769. https://doi.org/10.1016/j.foodhyd.2020.105769.

[17] R.A. De Freitas, T. Nicolai, C. Chassenieux, L. Benyahia, Stabilization of Water-inWater Emulsions by Polysaccharide-Coated Protein Particles, Langmuir. 32 (2016) 1227-1232. https://doi.org/10.1021/acs.langmuir.5b03761. 
[18] L.A. Bello-Pérez, P. Roger, B. Baud, P. Colonna, Macromolecular Features of Starches Determined by Aqueous High-performance Size Exclusion Chromatography, J. Cereal Sci. 27 (1998) 267-278. https://doi.org/10.1006/jcrs.1998.0186.

[19] A. Kharlamova, W. Inthavong, T. Nicolai, C. Chassenieux, The effect of aggregation into fractals or microgels on the charge density and the isoionic point of globular proteins, Food Hydrocoll. 60 (2016) 470-475.

https://doi.org/https://doi.org/10.1016/j.foodhyd.2016.04.013.

[20] E. Ben Ayed, R. Cochereau, C. Dechancé, I. Capron, T. Nicolai, L. Benyahia, Water-InWater Emulsion Gels Stabilized by Cellulose Nanocrystals, Langmuir. 34 (2018) 68876893. https://doi.org/10.1021/acs.langmuir.8b01239.

[21] V.T.N.T. Bui, B.T. Nguyen, T. Nicolai, F. Renou, Mixed iota and kappa carrageenan gels in the presence of both calcium and potassium ions, Carbohydr. Polym. 223 (2019) 115107. https://doi.org/https://doi.org/10.1016/j.carbpol.2019.115107.

[22] K.R. Peddireddy, T. Nicolai, L. Benyahia, I. Capron, Stabilization of Water-in-Water Emulsions by Nanorods, ACS Macro Lett. 5 (2016) 283-286.

https://doi.org/10.1021/acsmacrolett.5b00953.

[23] A. Gonzalez-Jordan, L. Benyahia, T. Nicolai, Cold gelation of water in water emulsions stabilized by protein particles, Colloids Surfaces A Physicochem. Eng. Asp. 532 (2017) 332-341. https://doi.org/10.1016/j.colsurfa.2017.04.073. 


\section{Supplementary Information}

\section{Effect of adding a third polysaccharide on the adsorption of protein microgels at the interface of polysaccharide-based water in water emulsions}

João P. E. Machado, Lazhar Benyahia and Taco Nicolai

not diluted

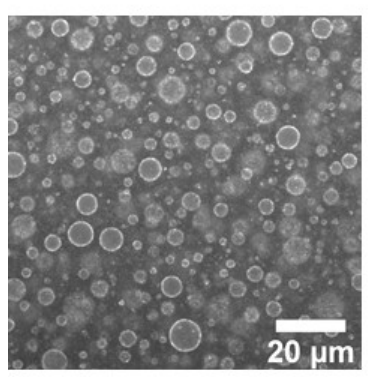

0.4

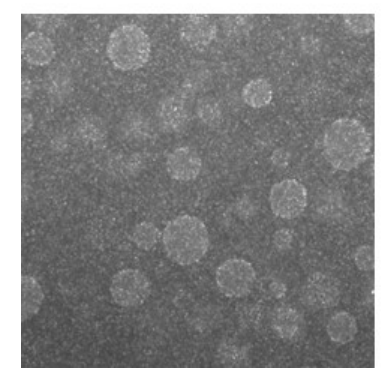

0.1

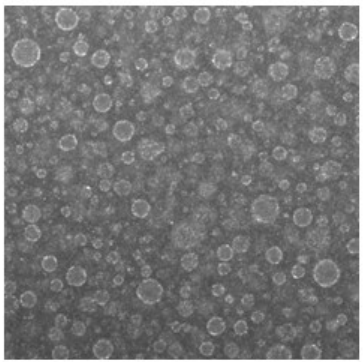

0.5

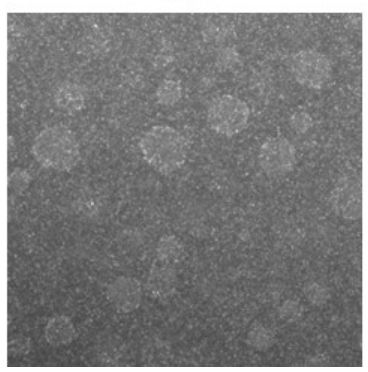

0.2

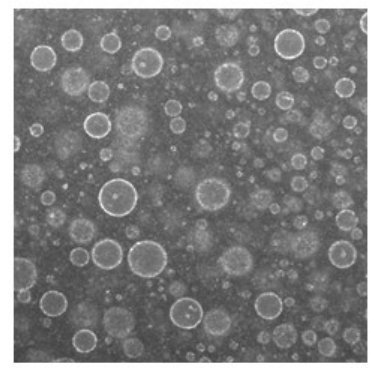

0.6

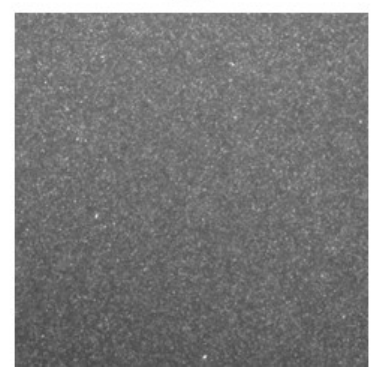

0.3

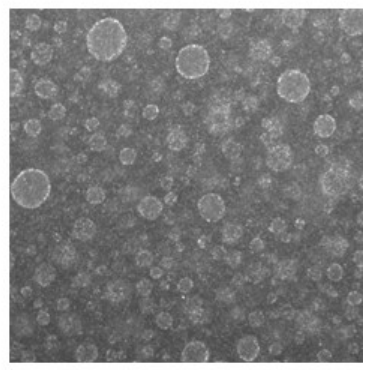

Fig. S1. CLSM images of A/P emulsions with 0.4 wt\% MG as a function of the dilution factor. All images are on the same scale. The composition of the undiluted emulsion was $1.2 \mathrm{wt} \% \mathrm{AMP}$ and 5.1 wt $\%$ PUL. 

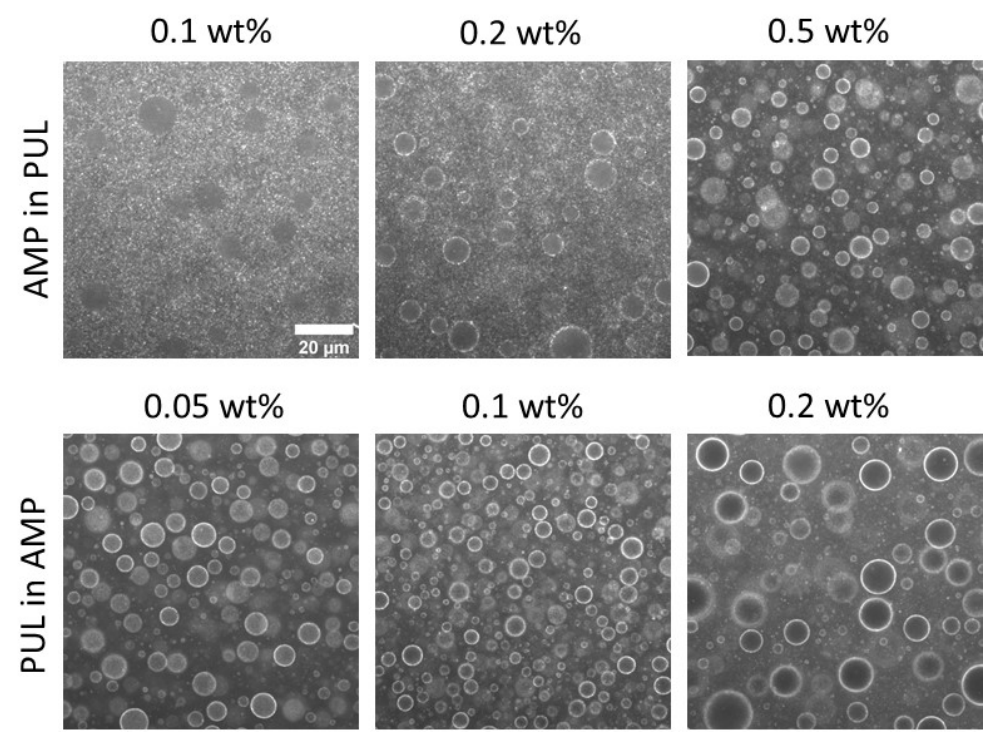

Fig S2. CLSM images of $A / P$ and $P / A$ emulsions with 0.4 wt\% $M G$ and different $X G$ concentrations. All images are on the same scale.

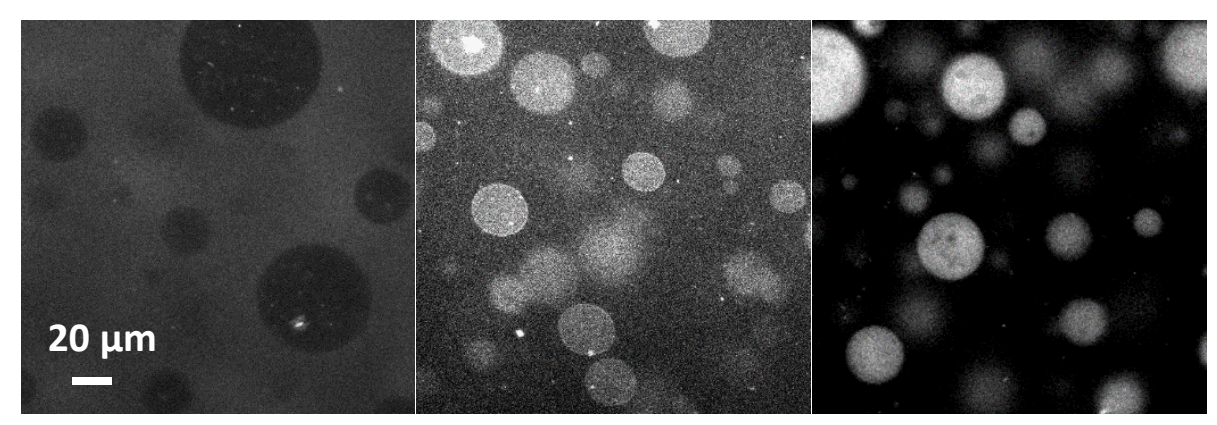

Fig. S3 CLSM images of A/P emulsions with 0.2 wt\% CNC without PEC (left), 0.11 wt\% PEC (middle) and $0.18 w t \%$ PEC (right). The contrast was enhanced to facilitate visualization. 